\section{Die Megastädte des Südens in der Geographie globaler Finanzmärkte und weltweiter Unternehmensnetzwerke}

\author{
von Christof Parnreiter, Universität Hamburg
}

\begin{abstract}
Anhand der Daten zur weltweiten Börsenkapitalisierung, zum Aktienhandel und zu den Standorten der größten Unternehmen wird gezeigt, dass sich die Geographie der Weltwirtschaft durch eine sehr starke Konzentration auf wenige Städte - allen voran New York, Tokio, London und Paris - auszeichnet. Die Megastädte Lateinamerikas und allgemeiner der Dritten Welt hingegen sind auf der Karte wirtschaftlicher Macht nur am Rande vermerkt. Das bedeutet aber nicht, dass sie für das Funktionieren von Globalisierungsprozessen unwichtig wären. Wie am Beispiel von Mexiko-City gezeigt wird, sind auch in Megastädten, die keine großen globalen Finanzplätze darstellen, wichtige Firmen der Unternehmensdienstleistungen angesiedelt, die von dort aus Dienste für die Organisation globaler Güterketten bereitstellen.
\end{abstract}

\section{Einleitung}

„Megastadt“ ist ein quantitativ bestimmter Begriff; derzeit liegt dafür der Schwellenwert bei einer Stadtbevölkerung von zehn Mio. Personen (UNPD 2006). Diese quantitative Bestimmung macht den Begriff ungeeignet für die Untersuchung qualitativer Veränderungen und Zusammenhänge, wie sie in der Analyse der Wechselwirkungen zwischen Globalisierungsprozessen und Stadtentwicklung angesprochen sind (Parnreiter 2007, Kap. 4.1). Nur manche Megastädte sind, wie in diesem Text gezeigt wird, zentrale Orte in der Geographie globaler Finanzmärkte und in weltweiten Unternehmensnetzwerken, während umgekehrt Städte zu den „Global Cities“ (Sassen 1991) gehören, die wiederum nicht zu den Megastädten zählen. Die meisten Megastädte Lateinamerikas, Afrikas und Asiens sind auf der Landkarte wirtschaftlicher Macht und Profite, wenn überhaupt, dann klein vermerkt.
Daraus zu schließen, dass die Megastädte der low and middle income countries für das Funktionieren der Weltwirtschaft weitgehend irrelevant wären, ist allerdings ein gravierender Fehler. Die Rolle, die Megastädten in Lateinamerika, Afrika oder Asien in globalen Produktionsnetzwerken zukommt, ist ein wenig erforschtes Feld. Konzeptionell spiegelt sich dieses Manko in der (auch in der deutschsprachigen Stadtgeographie weitverbreiteten) Trennung von Megastadt- und Global-CityForschung ebenso wider wie in einem skandalisierenden Alarmismus, für den das ebenso populäre wie populärwissenschaftliche Buch „Planet of Slums“ ein gutes Beispiel ist (Davis 2006). Empirisch zeigt sich, dass nur wenige AutorInnen sich an die Aufgabe gemacht haben zu analysieren, ob und welche wirtschaftlichen Tätigkeiten in den Megastädten des globalen Südens ausgeübt werden, die für die tägliche Praxis der Globalisierung essentiell sind. Um diesem Manko abzuhelfen, wird in diesem Text nach einer Skizze der Geographie globaler Finanzmärkte und weltweiter Unternehmensnetzwerke die Rolle von Mexiko-City als ,gateway“ aktueller Globalisierungsprozesse untersucht.

\section{Zur Geographie globaler Finanzmärkte und weltweiter Unternehmensnetzwerke}

Der globale Finanzmarkt ist stark auf wenige Städte konzentriert. An den New Yorker Börsen NYSE, Nasdaq und ASE wurden Ende $2008^{1} 36,3$ \% der weltweiten Börsenkapitalisierung erzielt, was die Stadt mit großen Abstand zum wichtigstem Finanzzentrum der Welt machte. Tokio als zweitwichtigster Standort kommt auf ein Viertel der Börsenkapitalisierung New Yorks, London auf ein Sechstel. Es folgen Shanghai, Hongkong, Mumbai, Toronto, Frankfurt, Zürich und Sydney. ${ }^{2}$ Drei Viertel der weltweiten Börsenkapitalisierung wird in diesen zehn Städten erzielt; vier davon sind Megastädte (New York, Tokio, Shanghai und Mumbai). Neben Shanghai und Mumbai hat nur noch eine Megastadt eines armen Landes einen nennenswerten Anteil an der weltweiten Börsenkapitalisierung, nämlich São Paulo (vgl. dazu Abb. 1 nächste Seite). 


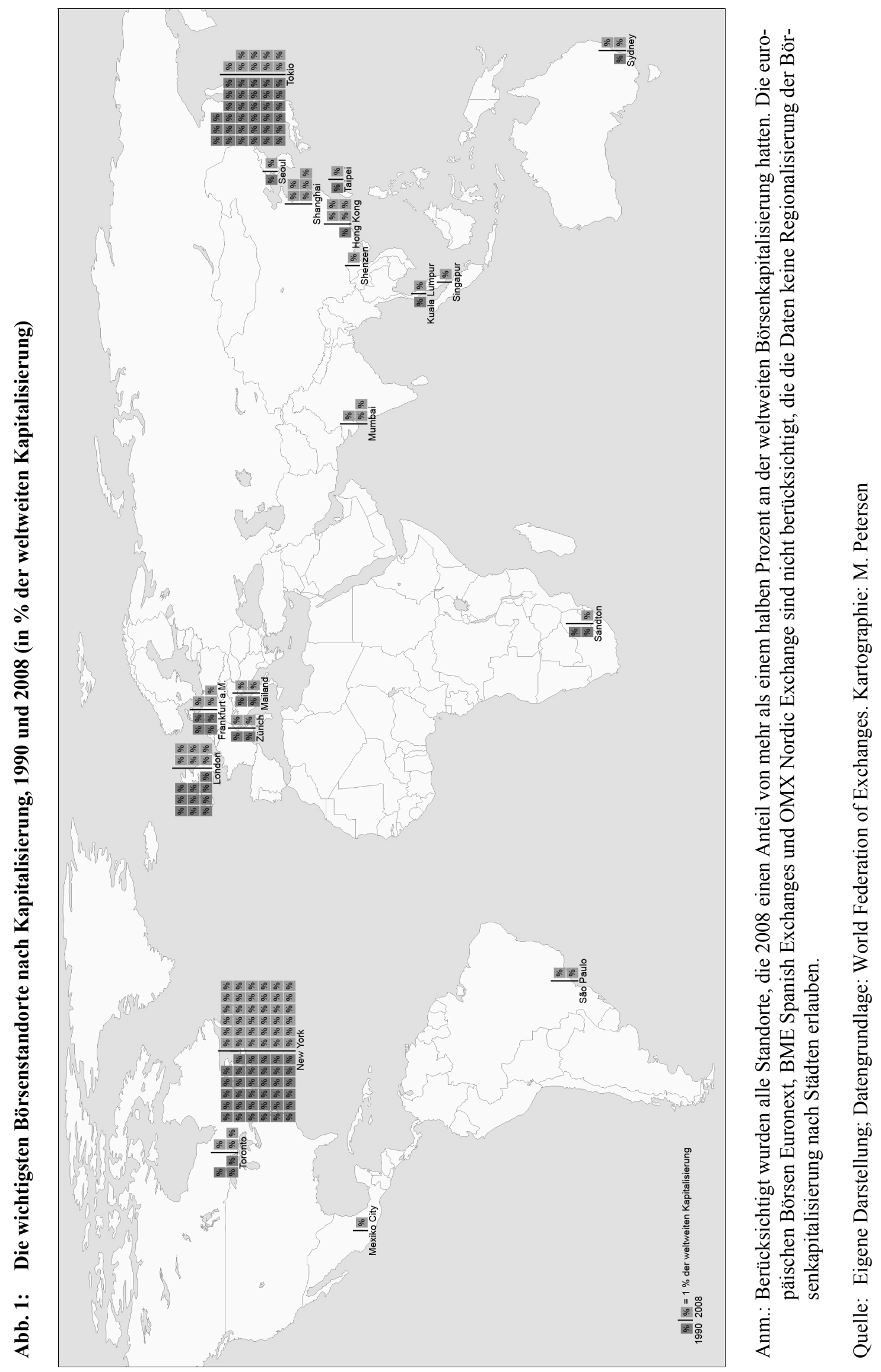


Eine Analyse der Entwicklung seit 1990 zeigt, dass der Anteil New Yorks an der gesamten Börsenkapitalisierung zwar schwankt (ein Höhepunkt wurde 2000 mit $49 \%$ erreicht), dass die Dominanz der Börsen dieser Stadt letztlich aber ungebrochen ist. Ein massiver Bedeutungsverlust ist aber für Tokio festzustellen, das, obwohl immer noch zweitwichtigster Börsenstandort, von fast einem Drittel der Kapitalisierung auf 9\% absackte. Auch London und Frankfurt erlitten, wenn auch in wesentlich geringerem Ausmaß, Anteilsverluste. Die Standorte, die an Bedeutung gewannen, liegen vorwiegend in Asien: Shanghai, Hongkong, Mumbai und Shenzen. Aber auch São Paulo und Mexiko-City spielten 1990 noch keine Rolle (vgl. Abb. 1).

Die historisch vergleichende Analyse erlaubt es auch, eine spezifische Geographie der massiven Entwertungen der auf den 56 Börsen der Welt notierten Unternehmen im Herbst 2008 zu erkennen. ${ }^{3}$ Zwischen Dezember 2007 und November 2008 sank ihr Börsenwert um 50,2\%; der Wertverlust betrug $31.500 \mathrm{Mrd}$. USD. Über ein Viertel dieser Entwertung fand in New York statt. Allerdings büssten die an New Yorker Börsen notierten Unternehmen unterdurchschnittlich, nämlich „nur“ 41,8 \% ihres Wertes, ein, weshalb der Anteil New Yorks an der weltweiten Börsenkapitalisierung zwischen Dezember 2007 und November 2008 auch stieg (von 31,8 auf 36,3\%). Die Krise des Finanzsystems im Jahr 2008 stärkte also „das Zentrum der Zentren“ - ein Aspekt, der es wert ist, künftig genauer analysiert zu werden. Auch Tokio und Zürich gingen relativ gesehen - gestärkt aus der Krise des Jahres 2008 hervor (mit Anteilssteigerungen von 7,1 auf 9,3\% bzw. von 2 auf 2,5\%), während London und Frankfurt einerseits, die Börsen Asiens und Lateinamerikas andererseits verhältnismäßig höhere Verluste verzeichneten. Vor allem in Shanghai und Mumbai, aber auch in São Paulo wurden die Werte der an den dortigen Börsen notierten Unternehmen überdurchschnittlich stark reduziert, während Mexiko-City relativ gesehen weniger betroffen war. Dies mag an der engen Koppelung der mexikanischen Wirtschaft an die USamerikanische liegen, müsste aber noch tiefer analysiert werden. Insgesamt verstärkte die Krise die zentripetalen Dynamiken auf den globalen Finanzmärkten (der Anteil der zehn wichtigsten Städte stieg zwischen Dezember 2007 und November 2008 von 71,6 auf 74,3 \%), während die Position der Ränder der Weltwirtschaft geschwächt wurde. ${ }^{4}$

Auch der Handel mit Aktien ist sehr stark auf New York konzentriert. Im Jahr $2008^{5}$ wurden $55 \%$ des weltweiten Aktienhandels an den drei New Yorker Börsen getätigt - fast acht Mal so viel wie in London, dem zweitwichtigsten Handelsplatz, und neun Mal so viel wie in Tokio. Ähnlich wie im Falle der Börsenkapitalisierung hat die Konzentration auf New York auch bezüglich des Aktienhandels seit 1990 zugenommen. Auch hier wurden die zentripetalen Kräfte im Zuge der massiven Entwertungen des Jahres 2008 gestärkt: New York erhöhte seinen Anteil am weltweiten Handel mit Aktien um fast zehn Prozentpunkte, während Tokio, London, Frankfurt sowie Shanghai und Shenzhen deutlich an Anteilen verloren. Von den Megastädten Afrikas, Asiens und Lateinamerikas haben nur Shanghai (2008: 2 \%), Mumbai und São Paulo (je $1 \%$ ) einen nennenswerten Anteil am weltweiten Aktienhandel; Mexiko-City, Buenos Aires und andere Megastädte der Peripherie hingegen sind im globalen Vergleich Handelsplätze ohne Bedeutung. ${ }^{6}$

Eine - vor den Bankenzusammenbrüchen des Herbstes 2008 durchgeführte - Analyse der Geographie der Unternehmenssitze der 75 größten Finanzdienstleister weltweit bestätigt die absolute Dominanz New Yorks in der Weltwirtschaft (Taylor et al. 2009). ${ }^{7}$ Es folgen London, Zürich, Paris, Toronto und - erst auf Rang 6 - Tokio. Unter den für den „Financial Command Index" wichtigsten 20 Städten findet sich neben New York und Tokio nur eine weitere Megastadt (Peking auf Rang 10), die „low and middle income countries" sind neben Peking noch mit St. Petersburg (Rang 18) vertreten. Shanghai, Mumbai und São Paulo, die als Börsenstandorte eine gewisse Bedeutung haben, sind als Unternehmenssitze für globale Finanzdienstleister hingegen weitgehend irrelevant.

Auch die Geographie weltweiter Unternehmensnetzwerke ist durch eine starke Zentralisierung wirtschaftlicher Macht geprägt. Um die Organisationslogiken der Weltwirtschaft zu analysieren, wird heute vermehrt auf 
nicht-staatszentrierte Konzepte wie „global commodity chains", „global value chains" oder „global production networks" zurückgegriffen. Ein Grundgedanke solcher Ansätze ist es, dass wirtschaftliche Aktivitäten vielfach in grenzüberschreitenden Produktionsketten bzw. -netzwerken organisiert sind. In der Herstellung von Gütern und Dienstleistungen werden so zahlreiche Orte in unterschiedlichen Ländern über die Flüsse von Kapital, Produkten, Informationen, Personen usw. miteinander verbunden. Ein zweiter Grundgedanke ist, dass solche dezentralen Produktionsketten bzw. -netzwerke nicht nur aus reinen Marktbeziehungen bestehen. Sie besitzen Governance-Strukturen, wobei Governance definiert wird als ,authority and power relationships that determine how financial, material and human resources are allocated and flow within a chain" (Gereffi 1994, S. 97). An anderer Stelle wurde darauf hingewiesen, dass diese Autorität lokalisiert werden kann, nämlich in Global Cities. Sie sind die Orte, an denen jene Inputs geleistet werden, die sowohl für das Funktionieren als auch für die Steuerung von Produktionsketten bzw. -netzwerken erforderlich sind (Parnreiter 2003, Parnreiter 2010, i. E.; Brown et al. 2007).

Abbildung 2 zeigt, dass die Firmensitze der weltgrößten Unternehmen stark konzentriert sind - auch wenn die Zentralisierung schwächer ist als bezüglich der Finanzmärkte. Die Hauptsitze der 500 größten Unternehmen der $\mathrm{Welt}^{8}$ verteilen sich auf 221 Städte, was zunächst als ein relativ dezentrales Muster erscheint. $^{9}$ Allerdings zeigt sich, dass die Hälfte der Umsätze „Big 500“ von Unternehmen gemacht werden, die in nur 20 Städten beheimatet sind. In Tokio finden sich die „headquarters” von 48 der 500 größten Unternehmen, die auf $8 \%$ der Umsätze der „Big 500 “ kommen. Es folgen Paris, London, New York und Peking. Bezogen auf die hier gestellte Frage der Megastädte und verglichen mit der Geographie der Finanzmärkte fällt auf, dass unter den 20 wichtigsten „HeadquarterStädten“ nur drei Megastädte sind (Tokio, New York und Peking), von denen zwei in „high income countries“ liegen. Insgesamt kommen Unternehmen, die in einer der 20 Megastädte beheimatet sind, auf $22 \%$ der Umsätze der Top-500-Unternehmen; die Megastädte Afrikas, Asiens und Lateinamerikas aber sind auf der Landkarte der wichtigsten Unternehmenssitze nur sehr randlich vermerkt. Außer in Peking finden sich Top-500Firmen noch in Moskau, Mexiko-City, Rio de Janeiro, São Paulo, Mumbai, New Delhi und Shanghai. Allerdings kommen die Firmen in diesen Städten zusammen auf nur 3,2 \% der Umsätze der „Big 500“, weshalb sie sich auch nicht auf der Karte der wichtigsten Unternehmensstandorte finden.

Untersucht man die 500 größten Unternehmen der Welt nach Gewinnen, so ergibt sich ein etwas anderes Bild. Zum einen sind die Gewinne noch stärker konzentriert als die Umsätze: In den 20 wichtigsten Städten finden sich die „headquarters“ von 214 Unternehmen, die zusammen $53 \%$ der Gewinne machen. Zum anderen ist bezüglich der Gewinne London das Zentrum - hier laufen die meisten Profite zusammen (7\%). Es folgen Paris, Tokio, New York und Peking (vgl. Abb. 2). Unter den 20 bezüglich der Gewinne von Unternehmen wichtigsten Städten finden sich fünf Megastädte, zu den genannten Tokio, New York und Peking kommen noch zwei aus ,middle income countries": Moskau und Rio de Janeiro. Auffällig ist auch, dass Unternehmen aus Megastädten in Afrika, Asien und Lateinamerika immerhin für $10 \%$ der Gewinne der „Big 500“ verantwortlich sind, wobei allerdings fast die Hälfte auf Peking und knapp $3 \%$ auf Moskau entfallen (weiters: Rio de Janeiro, Mumbai, Mexiko-City, Shanghai, São Paulo).

Wiederum zeigt die Analyse von Taylor et al. (2009) eine im Wesentlichen ähnliche, in einigen Punkten aber abweichende Geographie wirtschaftlicher Macht. Laut „Business Command Index ${ }^{\text {"10 }}$ ist Tokio das weltweit dominante Zentrum, gefolgt von New York, London, Paris und Houston. Weitere Megastädte unter den wichtigsten 20 Städten sind Osaka (Rang 8), Peking (9) und Los Angeles (12); Peking ist auch die einzige Stadt aus low and middle income countries, die sich in der Liste findet. 


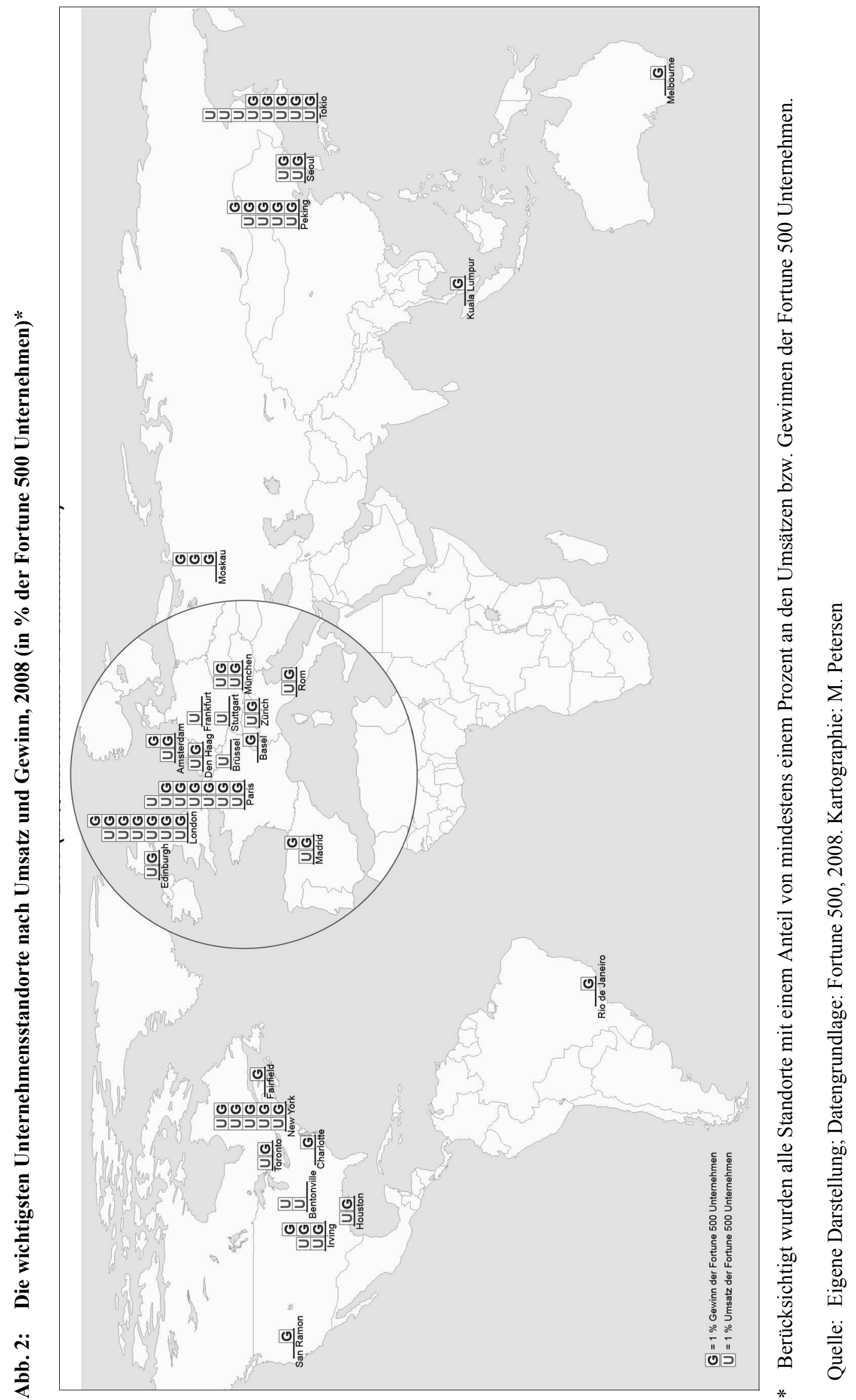


Zusammenfassend kann also hinsichtlich der hier gestellten Frage nach der Rolle der Megastädte in der Geographie der Weltwirtschaft festgehalten werden, dass New York, Tokio, London und Paris die globalen Zentren wirtschaftlicher Macht und Profite sind, während von den Megastädten Afrikas, Asiens und Lateinamerikas lediglich vier eine gewisse Bedeutung besitzen: Shanghai, Peking, Mumbai und - mit Abstand - São Paulo. ${ }^{11}$ Zwei der drei weiteren lateinamerikanischen Megastädte, nämlich Mexiko-City und Rio de Janeiro, sind in der Geographie globaler Finanzmärkte und „headquarters” als „Dörfer“ verzeichnet, Buenos Aires kommt gar nicht vor.

Kann sich eine Untersuchung der Rolle von Megastädten in globalen Finanzmärkten bzw. in der Weltwirtschaft insgesamt aber auf die Analyse von Finanzmärkten oder von Unternehmensansiedlungen beschränken? Gibt es nicht jenseits der Börsen und Konzernzentralen ökonomische Tätigkeiten, die für das Funktionieren von Globalisierungsprozessen wichtig sind, und die sich dann (vielleicht) doch auch in den Megastädten Afrikas, Asiens und Lateinamerikas finden? Diesen Fragen, die in der Forschung über Megastädte gegenüber Themen wie Risikoexponiertheit oder sozialer wie ökologischer Probleme deutlich unterprivilegiert sind, soll abschließend am Beispiel von Mexiko-City nachgegangen werden. ${ }^{12}$

\section{Mexiko-City als ,gateway“ aktueller Globalisierungsprozesse}

Mexiko-City kommt, wie erwähnt, in der Geographie globaler Finanzmärkte und ,headquarters" nur am Rande vor. Zugleich aber wurde Mexiko in den letzten zweieinhalb Jahrzehnten immer stärker in globale Güterketten eingebunden. Die jährlichen Zuflüsse von ausländischen Direktinvestitionen (ADI) wuchsen um $1.300 \%$ (1980/85 - 2001/06), um sich 2006 auf 19 Mrd. USD zu belaufen, was Mexiko hinter China zum zweitwichtigsten Empfänger von ADI in den ,low and middle income countries” machte (UNCTAD 2007). Auch die Ex- und Importe nahmen drastisch zu: Während die jährlichen Ausfuhren verneunfacht wurden $(1980 / 85 \text { - 2002/07) })^{13}$, wuchsen die Importe auf das Zwölffache an; die Außenhandelsquote stieg von $24 \%$ in den frühen 1980er Jahren auf
$65 \%$ (2006) an (UNCTAD 2008; World Bank 2009). Mindestens ebenso bedeutsam ist, dass sich die Zusammensetzung der Exporte veränderte: Industrieexporte machen inzwischen $80 \%$ der Ausfuhren aus (2006-08), Erdöl kommt nur noch auf $17 \%$ (INEGI 2009).

Mexiko wurde also zu einer Plattform der Exportproduktion für den US-Markt gemacht. Das wirft die Frage auf, von wo aus die Produktion entlang der mexikanischen Abschnitte der globalen Fließbänder gemanagt wird. Die im globalen Vergleich der Standorte für Börsenhandel und Unternehmenssitze gesehene Bedeutungslosigkeit von Mexiko-City führt vielfach zur falschen Einschätzung, dass diese Stadt (gleich wie viele andere Megastädte in Afrika, Asien und Lateinamerika) funktional irrelevant wäre für das Management von Globalisierungsprozessen. Tatsächlich aber ist Mexiko-City ein Ort, von dem aus notwendige Inputs bereitgestellt werden. Nach Sassen sind das ,,specialized services needed by complex organizations for running a spatially dispersed network of factories, offices, and service outlets" (Sassen 1991, S. 5). Indem solche hochwertigen Dienstleistungen in globale Güterketten eingespeist werden, übernimmt Mexiko-City Global-City-Funktionen. ${ }^{14}$ Eine ähnliche Transformation ist für Megastädte wie São Paulo, Mumbai, Shanghai oder auch Moskau sehr wahrscheinlich, im Detail aber bislang wenig erforscht worden.

Die Bedeutung von Mexiko-City für das Management von Globalisierungsprozessen zeigt sich an der extrem hohen Zentralisierung der Unternehmensdienste, der Konzernzentralen und der Finanzflüsse. Während in den Städten, in denen die Weltmarktproduktion stattfindet und die zu einem erheblichen Teil an der Nordgrenze Mexikos liegen, so gut wie keine Unternehmensdienste hergestellt werden, konzentriert Mexiko-City die Wertschöpfung in diesem Bereich: $76 \%$ der in Mexiko erzielten Wertschöpfung in Unternehmensdienstleistungen kommen aus Mexiko-City, bei Finanzdiensten sind es gar $88 \%$. Eine Analyse der Standortpräferenzen der 500 größten in Mexiko registrierten Unternehmen stützt die These von der Global-CityFormation weiter. Seit 1993, dem Jahr bevor das Nordamerikanische Freihandelsabkommen „NAFTA“ in Kraft trat, hat die Konzentration der ,headquarters” auf Mexiko-City 
zugenommen - 2006 hatten 352 der Top 500 dort ihren Firmensitz. Bei Unternehmen im ausländischen Besitz ist der Anteil noch höher - $80 \%$ sind in Mexiko-City niedergelassen. Schließlich verbucht Mexiko-City mit etwa zwei Dritteln den Löwenanteil an den ausländischen Direktinvestitionen $\left(1994\right.$ - 2008) ${ }^{15}$

Wie wichtig Mexiko-City für das Funktionieren globaler Produktionsketten ist, zeigt sich auch an der hohen Präsenz weltweit operierender Unternehmensdienstleister, was hier am Beispiel der Buchhaltungs- und Beratungsfirmen verdeutlicht werden soll. Die „Big Four“ dieser Branche (Deloitte, Ernst \& Young, KPMG, PricewaterhouseCoopers) unterhalten große Büros in Mexiko-City, zu ihren Kunden zählen die wichtigsten Unternehmen in Mexiko. Von den an der mexikanischen Börse gelisteten Firmen ${ }^{16}$ nehmen $91 \%$ die Dienste eine der vier genannten Beratungsfirmen in Anspruch; von den 100 größten Unternehmen haben nur drei eine nicht-globale Buchhaltungsfirma. Das bedeutet, dass zahlreiche durch Mexiko laufende oder dort entspringende Güterketten Dienste aus Mexiko-City beziehen, die dort von global operierenden Firmen bereitgestellt werden: Der staatliche Ölkonzern PEMEX wird ebenso wie die private Firma CEMEX, der weltweit drittgrößte Zementhersteller, von KPMG beraten, während Teléfonos de México mit der Tochtergesellschaft América Móvil, Lateinamerikas größter Mobiltelefonanbieter, Buchhaltungsund andere Dienste ebenso von Ernst \& Young beziehen wie z. B. Wal-Mart. ${ }^{17}$ In der Autoindustrie, einem der wichtigsten Exportsektoren Mexikos, arbeitet Deloitte für General Motors, PricewaterhouseCoopers für Volkswagen und Ford, KPMG für Chrysler und Ernst \& Young für Nissan. Die Knotenfunktion von MexikoCity ist aber nicht auf Unternehmensberatung und Buchhaltung beschränkt. Die Analysen der „Globalization and World Cities Study Group and Network" zeigen, dass Mexiko-City eine insgesamt hohe Bedeutung in den weltweitweiten Netzwerken global operierender Firmen des Unternehmensdienstleistungssektors hat, weshalb für Taylor Mexiko-City auch ,a classic gateway [city] of contemporary globalization“" darstellt (Taylor 2004, S. 92).

\section{Schnittstelle im Werttransfer oder Motor nachhaltiger Entwicklung?}

Der Begriff ,gateway“ hat allerdings mehrere Bedeutungen. Wird er als „Schnittstelle“ übersetzt, so bedeutet das, dass Mexiko-City ein Scharnier darstellt - einen Ort, an dem Unternehmen wie die „Big Four“ der Buchhaltungsund Unternehmensberatungsfirmen, aber auch zahlreiche Finanzdienstleister oder global tätige Anwaltskanzleien die Produktion in Mexiko mit dem Weltmarkt vernetzen. Gateway kann aber auch als „Einfallstor“ übersetzt werden, und in diesem Kontext schließt der Begriff an frühe dependenztheoretische Arbeiten an. André Gunder Frank konzeptionalisierte die Metropolen Lateinamerikas als Brückenköpfe des „Nordens“, die notwendig sind, um den Werttransfer aus dem Süden zu organisieren: „Just as the colonial and national capital (...) become the satellite of the Iberian (and later of other) metropoles of the world economic system, this satellite immediately becomes a colonial and then a national metropolis with respect to the productive sectors and population of the interior. (...) Thus, a whole chain of constellations of metropoles and satellites relates all parts of the whole system from its metropolitan center in Europe or the United States to the farest outpost in the Latin American countryside." (Frank 1969, S. 6) Folgt man dieser Analyse, so sind Megastädte wie Mexiko-City zwar keine globalen Finanzzentren, wohl aber strategisch wichtige Orte, um weltweit ungleicher Entwicklung zu organisieren.

Wenigstens für Lateinamerika lässt sich damit auch die Frage beantworten, welche Rolle Megacities für eine nachhaltige Entwicklung spielen. Im Zuge der wirtschaftlichen Reorientierung auf den Weltmarkt, also der Öffnung für Importe und Kapitalflüsse sowie der Ausrichtung der Produktion auf Exportmärkte, haben die Ökonomien von Städten wie Mexiko-City ihre Fähigkeit, den nationalen Binnenmarkt zu integrieren, weitgehend verloren (Parnreiter 2007). ${ }^{18}$ Alle verfügbaren Daten weisen darauf hin, dass - verglichen mit den Zeiten der Importsubstitution - das wirtschaftliche Wachstum sich ebenso verlangsamt hat wie die Kapazität, neue Arbeitsplätze zu schaffen. Die realen Löhne fallen oder stagnieren, während verschiedene Formen informeller Beschäftigung deutlich zunehmen. Wie wenig nachhaltig der Entwick- 
lungsweg der Transformation der lateinamerikanischen Megastädte hin zu Global Cities ist, zeigt sich auch daran, dass es seit den 1980er Jahren zu einer Verstädterung der Armut gekommen ist: Mehr als zwei Drittel der Armen Lateinamerikas leben heute in den Städten.

\section{Anmerkungen}

1) Stand November.

2) Euronext kommt auf 6,2\% der weltweiten Kapitalisierung, BME Spanish Exchanges auf 2,8 \% und OMX Nordic Exchange auf 1,7\%. $\mathrm{Da}$ es sich in diesen Fällen aber um Zusammenschlüsse einzelner Börsen handelt (nämlich von Amsterdam, Brüssel, Lissabon und Paris bei Euronext, von Barcelona, Bilbao, Madrid und Valencia bei BME Spanish Exchanges und von Kopenhagen, Helsinki, Island, Stockholm, Tallinn, Riga und Vilnius bei OMX Nordic Exchange), ist eine präzise Regionalisierung der Börsenkapitalisierung nach Städten nicht möglich, weshalb die genannten Börsen hier nicht berücksichtigt werden.

3) Die Krise war beim Verfassen dieses Textes keineswegs überwunden, weshalb die Aussagen hier lediglich eine Zwischenbilanz darstellen.

4) Dieser Beobachtung liegen eigene Berechnungen zu Grunde, die auf World Federation of Exchanges aus dem Jahr 2009 zurückgehen.

5) Stand November.

6) Dieser Beobachtung liegen eigene Berechnungen zur Grunde, die auf World Federation of Exchanges aus dem Jahr 2009 zurückgehen.

7) Die Analyse beruht auf der Forbes-Liste der 2.000 größten Unternehmen weltweit, die verschiedene Indikatoren kombiniert (Umsätze, Gewinne, Marktwert und Vermögenswerte).

8) Stand 21.7.2008.

9) Dieser Beobachtung liegen eigene Berechnungen zur Grunde, die auf Fortune 500 (2009) zurückgehen.

10) Siehe Anmerkung 7.

11) $\mathrm{Zu}$ den Ursachen der starken Zentralisierung der Finanzmärkte und der Unternehmenszentralen vgl. z. B. Sassen 1991.

12) Ausführlichere Hinweise dazu finden sich in Parnreiter 2010 / i. E.

13) Um Verfälschungen zu vermeiden, wurden bei dieser Berechnung für die beiden Vergleichzeiträume jeweils der Durchschnitt über die Werte der Einzeljahre gebildet und diese anschließend verglichen.

14) Siehe dazu Kapitel 5 in Parnreiter 2007.

15) Da die Daten auf Bundesstaatsebene publiziert werden, sind genaue Angaben für die Stadt nicht möglich; der „Distrito Federal“, der innere Teil Mexiko-City's, kommt auf $57 \%$. Die hier verwendeten Aussagen stützen sich auf eine Reihe verschiedener Quellen: INEGI 2004; „Expansión“ (verschiedene Jahrgänge); Secretaría de Economía 2009).

16) 220 der 300 größten Unternehmen in Mexiko sind börsennotiert.

17) Bei Wal-Mart ist bemerkenswert, dass das Unternehmen für die schnelle Globalisierung des mexikanischen Einzelhandelssektors verantwortlich ist.

18) Santiago de Chile stellt für viele der Negativtrends eine Ausnahme dar, fällt allerdings auch nicht in die Klassifizierung „Megastadt“.

\section{Literatur}

Brown, E.; Derudder, B.;Parnreiter, C. et al., 2007: World City Networks and Global Commodity Chains: Towards a World-Systems' Integration. GaWC Research Bulletin 236. Loughborough; http://www.lboro.ac.uk/gawc/rb/rb236.html (download 16.1.09)

Davis, M., 2006: Planet of Slums. New York

Expansión, verschiedene Jahre: Las 500 empresas más importantes de México. México D.F.

Fortune 500, 2009: Global 500. Our annual ranking of the world's largest corporations. New York; http://money.cnn.com/magazines/fortune/global500/ 2008/full_list/ (download 16.1.09)

Frank, A.G., 1969: Latin America: Underdevelopment or Revolution: Essays on the Development of Underdevelopment and the Immediate Enemy. New York

Gereffi, G., 1994: The Organization of BuyerDriven Global Commodity Chains: How U.S. Retailers Shape Overseas Production Networks. In: Gereffi, G.; Korzeniewicz, M. (Hg.): Commodity Chains and Global Capitalism. Westport, S. 95-122

INEGI - Instituto Nacional de Estadísticas y Geografia, 2004: Censos Económicos 2004, Aguascalientes: Instituto Nacional de Estadísticas, Geografía e Informatica. Aguascalientes.

INEGI - Instituto Nacional de Estadísticas y Geografía, 2009: Banco de Información Económica, Instituto Nacional de Estadísticas, Geografía e Informatica. Aguascalientes; http://www.inegi.gob.mx (download 13.1.09)

Parnreiter, C., 2003: Global City Formation in Latin America: Socioeconomic and Spatial Transformations in Mexico City and Santiago de Chile. Paper presented at the 99th Annual Meeting of the Association of American Geographers, 4-8 March 2003. New Orleans; http://www.lboro.ac.uk/gawc/rb/rb103.html (download 16.1.09) 
Parnreiter, C., 2007: Historische Geographien, verräumlichte Geschichte. Mexiko-City und das mexikanische Städtenetz von der Industrialisierung bis zur Globalisierung. Stuttgart

Parnreiter, C., 2010 / i. E.: Global Cities in Global Commodity Chains. Towards a Geography of Governance in the World Economy. In: Global Networks, Special Issue

Sassen, S., 1991: The Global City. New York

Secretaría de Economía, Dirección General de Inversión Extranjera, 2009: Reporte de Estadísticas. México DF; http://www.si-rnie.economia.gob. $\mathrm{mx} /$ cgi-bin/repie.sh/reportes/selperiodo (download 25.1.09)

Taylor, P., 2004: World City Network. A Global Urban Analysis. London

Taylor, P.; Ni, P.; Derudder, B. et al., 2009: The Way We Were: Command-and-Control Centres in the Global Space-Economy on the Eve of the 2008 Geo-Economic Transition. In: Environment and Planning A, 41 (2009), S. 7-12

UNCTAD - United Nations Conference on Trade and Development, 2007: World Investment Report 2007. Transnational Corporations, Extractive Industries and Development. New York

UNCTAD - United Nations Conference on Trade and Development, 2008: Handbook of Statistics 2008. Genf; http://stats.unctad.org/handbook/Report Folders/ReportFolders.aspx?CS_referer $=\& C S \_C h o$ senLang=en (download 9.9.08)

UNPD - United Nations Population Division, 2006: World Urbanization Prospects. The 2005 Revision. New York

World Bank, 2009: World Development Indicators Quick Query. New York; http://ddp-ext.world bank.org/ext/DDPQQ/member.do?method=getMem bers\&userid=1\&queryId=135 (download 15.1.09)

World Federation of Exchanges, 2009: Domestic Market Capitalization. Paris; http://www.worldexchanges.org/statistics/annual/2004/domesticmarket-capitalization-1 (download 16.1.09)

\section{Kontakt}

Prof. Dr. Christof Parnreiter

Universität Hamburg

Institut für Geographie

Bundesstraße 55 (Geomatikum), 20146 Hamburg

Tel.: +49 (0) 40 / $42838-4961$

Fax: +49 (0) 40 / 42838 - 4967

E-Mail: parnreiter@geowiss.uni-hamburg.de

\section{Die HGF-Forschungsinitiative „Risk Habitat Megacity“: Ziele, Ansatz, Fragestellungen}

\author{
von Jürgen Kopfmüller, Helmut Lehn, ITAS, \\ sowie Dirk Heinrichs, Henning NuissI und \\ Kerstin Krellenberg, UFZ Leipzig
}

\begin{abstract}
Mega-Urbanisierung spielt aufgrund der Geschwindigkeit und des räumlichen AusmaBes sowie aufgrund ihrer weltweiten Auswirkungen eine entscheidende Rolle für die nachhaltige Entwicklung unseres Planeten. Besonders die großen Agglomerationen nehmen in diesem Prozess eine doppelte Rolle ein: Sie sind Täter und Opfer zugleich. Vor diesem Hintergrund werden Ziele und Untersuchungsansatz der Helmholtz-Forschungsinitiative „Risk Habitat Megacity“" skizziert und die Analysen am Beispiel zweier Anwendungsfelder (,,Sozialräumliche Differenzierung“ und „Wasser - Ressourcen und Dienstleistungen") illustriert.
\end{abstract}

\section{Warum Forschung für Megastädte?}

Mega-Urbanisierung spielt aufgrund der Geschwindigkeit und der räumlichen Wirkungen sowie aufgrund ihrer Rolle in Prozessen des globalen Wandels eine entscheidende für die Zukunft unseres Planeten. Sie stellt einerseits eine treibende Kraft des globalen Wandels dar; andererseits akkumulieren sich dessen Auswirkungen gerade in den Megastädten, so dass diese zugleich „Täter“ und „Opfer“ (potenziell) krisenhafter und riskanter Erscheinungsformen des globalen Wandels sind.

Die Erforschung megaurbaner Entwicklungsprozesse und ihrer Wechselwirkungen gehört daher zu den großen wissenschaftlichen Herausforderungen unserer Zeit:

- Die rasante Entwicklung von Megastädten und Metropolen ist in ihren regionalen und globalen Folgen bislang noch wenig erforscht. Neu sind nicht nur die enorme Konzentration von Bevölkerung, Infrastruktur und Wirtschaftskraft an einem Ort, sondern auch die Gleichzeitigkeit und Komplexität verschiedenster Prozesse. Sie reichen von der Verschärfung sozialer Gegen- 